\title{
Anticholinergic burden and dry mouth among Finnish, community-dwelling older adults
}

Antti Tiisanoja $^{1,2}$, Anna-Maija Syrjälä ${ }^{1,2,3}$, Kaija Komulainen ${ }^{4,5}$, Pasi Lampela ${ }^{6,7}$, Sirpa

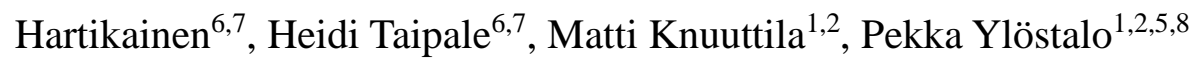

Running title: ADS and dry mouth in older people

Keywords: Aged, anticholinergic drugs, anticholinergic burden, Anticholinergic Drug Scale, dry mouth, hyposalivation, xerostomia,

${ }^{1}$ Unit of Oral Health Sciences Research, University of Oulu

${ }^{2}$ Medical Research Center Oulu, Oulu University Hospital and University of Oulu, Oulu

${ }^{3}$ Dental training clinic, Social and Health services, City of Oulu, Oulu, Finland

${ }^{4}$ Social and Health Centre of Kuopio, Kuopio, Finland

${ }^{5}$ Institute of Dentistry, University of Eastern Finland, Kuopio, Finland

${ }^{6}$ Kuopio Research Center of Geriatric Care, University of Eastern Finland, Kuopio, Finland

${ }^{7}$ Department of Social Pharmacy, School of Pharmacy, University of Eastern Finland, Kuopio, Finland

${ }^{8}$ Department of Oral and Maxillofacial Diseases, Kuopio University Hospital, Kuopio, Finland

Corresponding author:

Antti Tiisanoja

Periodontology and Geriatric Dentistry

Unit of Oral Health Sciences Research

P.O. BOX 5281

FI-90401, University of Oulu

Finland

antti.tiisanoja@student.oulu.fi 


\section{Abstract}

Objective: The aim was to study whether the anticholinergic burden of drugs is related to xerostomia and salivary secretion among community-dwelling elderly people.

Background: Anticholinergic drugs have been shown to be a risk factor for dry mouth, but little is known about the effects of cumulative exposure to anticholinergic drugs measured by anticholinergic burden on salivary secretion or xerostomia.

Methods: The study population consisted of 152 community-dwelling, dentate, non-smoking, older people from the Oral Health GeMS study. The data were collected by interviews and clinical examinations. Anticholinergic burden was determined by using the Anticholinergic Drug Scale (ADS). A Poisson regression model with robust error variance was used to estimate relative risks (RR) with 95\% confidence intervals (CI 95\%).

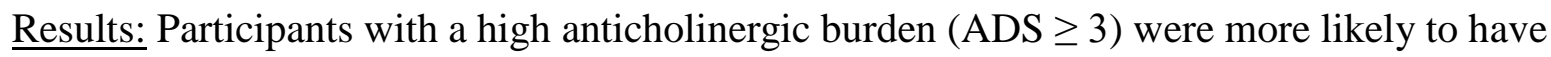
xerostomia (RR: 3.17; CI: 1.44-6.94), low unstimulated salivary flow (<0.1 ml/min; RR: 2.31, CI: 1.21-4.41), and low stimulated salivary flow (<1.0 ml/min; RR: 1.49, CI: 0.79-2.79) compared to reference group (ADS 0). In participants with a moderate anticholinergic burden (ADS 1-2) all the risk estimates for xerostomia, unstimulated and stimulated salivary secretion varied between $0.55-$ 2.85. Additional adjustment for the total number of drugs, anithypertensives, and sedative load caused only slight attenuation of the risk estimates.

Conclusion: A high anticholinergic burden was associated with low unstimulated salivary secretion and xerostomia. 


\section{$\underline{\text { Introduction }}$}

Nowadays dry mouth can be seen to consist of two conditions, xerostomia and hyposalivation ${ }^{1}$. Xerostomia is a subjective feeling of dry mouth, whereas hyposalivation is a condition with objectively lowered salivary output in either stimulated or unstimulated salivary secretion. The most important etiological factors for dry mouth among older people are drugs — especially anticholinergic drugs - and polypharmacy ${ }^{1,2}$.

Anticholinergic drugs include several drugs from different therapeutic categories ${ }^{3,4}$. Certain drugs, such as atropine or oxybutin, are used specifically because of their anticholinergic properties, while other drugs, such as antidepressants, antipsychotics, and antihistamines, have anticholinergic activities that are not necessarily related to their therapeutic effects. Despite the fact that anticholinergic drugs are associated with serious adverse events, they are commonly used among

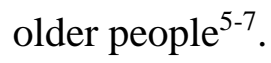

Previous studies have shown that drug-induced anticholinergic activity is an additive effect and that there is variability in the anticholinergic potency of drugs ${ }^{8,9}$. Therefore, several methods have been developed to measure an overall anticholinergic burden caused by drugs used ${ }^{10}$. One of these methods is Carnahan's Anticholinergic Drug Scale (ADS) ${ }^{11}$, which ranks drugs into four categories based on their anticholinergic activity and it takes into account the dose of anticholinergic drugs. An individual's anticholinergic burden is obtained by summing the categorical values of the drugs used. A benefit of using the anticholinergic burden is that it enables the focus to be on the overall anticholinergic effect of all the drugs used instead of just on certain specific drugs with a prominent anticholinergic effect.

It is well known that anticholinergic drugs cause hyposalivation and xerostomia, either by directly affecting muscarinic receptors in the salivary glands or by a secondary effect through the central nervous system ${ }^{9,10}$. Yet, little is known about the association between anticholinergic burden caused 
by all the drugs used and salivary secretion or xerostomia. A study by Kersten et al. ${ }^{11}$ showed, that among nursing home residents, a high anticholinergic burden (ADS $\geq 6$ ) was associated with low unstimulated salivary secretion. A high anticholinergic burden measured by another anticholinergic scale, the Anticholinergic Risk Scale, have also been previously reported to be associated with an increased risk of peripheral anticholinergic adverse effects, including xerostomia ${ }^{12}$.

Because of the high frequency of anticholinergic drug use among older people and the additive effect of these drugs, it is important to study the effects of overall anticholinergic burden on oral health. The aim of this study was to examine whether anticholinergic burden, measured by the ADS, is related to xerostomia, unstimulated, and stimulated salivary secretion among communitydwelling elderly people.

\section{$\underline{\text { Material and methods }}$}

Our study population is part of a larger Oral Health Geriatric Multidisciplinary Strategy for the Good Care of the Elderly (GeMS) study. The randomly selected participants were 75 year old or older on the first of November in 2003 and lived in the city of Kuopio. The Oral Health GeMS study included 354 people who underwent comprehensive geriatric assessments and oral examinations at the base line of the study. In the present study, the study population was further restricted to community-dwelling, non-smoking, dentate people (at least one clinically visible tooth or dental root), whose salivary secretion was measured, $n=152$ (109 women and 47 men, mean age 79.4, SD 3.67).

Written informed consent was provided by the participants or their close relatives or caregivers. The study protocol was approved by the ethics committee of Kuopio University Hospital and the University of Kuopio. Further information about the GeMS study can be found from previous studies $^{13-15}$.

A multidisciplinary team (two nurses, two physiotherapists and two physicians) carried out the 
comprehensive geriatric assessment, which included interviews and clinical examinations. In situations where the participant could not answer the questions because of his/her cognitive or other impairment, a close relative or a caregiver provided the answers. If the participant was unable to visit the health centre, the interviewer, accompanied by a physician, made a home visit to conduct the interview and clinical examination. The participant's self-reported drug use, both prescription and non-prescription drugs, was verified from prescription forms and drug packages. Additional health data were also taken from the medical records of local municipal health centres, home nursing services, local hospitals, and the Kuopio University Hospital.

Oral examinations of the participants of the Oral Health GeMS study were carried out by two dentists in 2004-2005, an average six months after the comprehensive geriatric assessment. The clinical oral examinations were done in the primary care setting of the Social and Health Centre of Kuopio or in the participant's home. During the examinations a WHO colour-coded periodontal probe, a mouth mirror and a gauze pad were used. The dentists performed the first seven examinations together in order to be calibrated. Since the examination was time-consuming (one hour) and caused strain to the participants, there were no repeated examinations.

\section{Outcome variables}

Salivary secretion, both stimulated and unstimulated, and xerostomia were used as outcome variables. All the participants answered questions about xerostomia and provided saliva samples. They were asked to abstain from eating or drinking one hour prior to the saliva collection. Participants with removable dentures gave an unstimulated saliva sample without the dentures and a stimulated saliva sample with the dentures.

The unstimulated saliva samples were collected using the draining method ${ }^{16}$. The participants were instructed to sit straight and bend their head forward. They were asked to swallow to empty their mouth and then to start drooling into a centrifuge tube for 5 minutes. The stimulated saliva samples 
were collected after the participants chewed a paraffin capsule for 30 seconds and emptied their mouth of the first saliva. Then the participants were asked to keep chewing the paraffin capsule for 5 minutes and drain the saliva into a centrifuge tube.

The stimulated salivary secretion was classified into two categories: $<1.0 \mathrm{ml} / \mathrm{min}$ (low) and $\geq 1.0$ $\mathrm{ml} / \mathrm{min}$ (normal) and the unstimulated salivary secretion also into two categories: $<0.1 \mathrm{ml} / \mathrm{min}$ (low) and $\geq 0.1 \mathrm{ml} / \mathrm{min}$ (normal). The cut-off values used are based on the literature ${ }^{17}$.

Xerostomia was determined with a single-item question 'How often does your mouth feel dry?' ${ }^{18}$. Xerostomia was classified into two categories based on the reported frequency of feeling of dry mouth: never/occasionally $v s$. often.

\section{Explanatory variable}

The anticholinergic burden caused by drugs was measured using Carnahan's Anticholinergic Drug Scale $^{8}$. The scale has been validated among a group of older long-term care residents and it classifies drugs into four score categories based on their anticholinergic activity, as follows:

- Score category 0 includes drugs with no known anticholinergic activity

- Score category 1 includes drugs with potential anticholinergic activity as evidenced by receptor-binding studies (e.g., furosemide, digoxin or captopril)

- Score category 2 includes drugs that are sometimes noted to have anticholinergic adverse events, usually with excessive doses (e.g., carbamazepine, cyproheptadine or disopyramide)

- Score category 3 includes drugs with marked anticholinergic activity (e.g., amitriptyline, brompheniramine or oxybutynin)

To determine each participant's final ADS score, dose-adjustments were done for drugs belonging to categories 2 and 3 and then the scores of all the drugs used were summed. This ADS score reflected anticholinergic burden and was classified into three categories: none (ADS 0), moderate (ADS 1-2) and high (ADS $\geq 3$ ). 


\section{Other variables}

The participant's sedative load was determined using the Sedative Load Model where each drug was placed into one of four categories based on the drug's sedative properties, and each category was assigned a sedative rating ${ }^{21,22}$.

- Sedative rating 0 was given to drugs with sedation as a potential but rare adverse reaction (e.g., second-generation antihistamines, acetylcholinesterase inhibitors) and to all other drugs with no known sedative properties

- Sedative rating 1 was given to drugs with sedation as a prominent side effect or drugs with a sedating component (e.g., SSRIs, atypical psychotics)

- Sedative rating 2 was given to first-line sedative drugs (e.g., anxiolytics, hypnotics)

To determine the participant's sedative load, all the sedative ratings of the regularly used drugs were summed.

Use of antihypertensive drugs was determined from the drug information and the following drug groups were included: $\mathrm{Ca}^{2}$-channel blockers, angiotensin-converting-enzyme inhibitors, $\mathrm{AT}_{1}$ receptor antagonists, diuretics, and selective $\beta$-blockers. For analyses, the use of antihypertensives was classified into two categories: no vs. yes.

Functional capability was determined by using Lawton's Instrumental Activities of Daily Living (IADL) scale ${ }^{19}$. The scale is based on a questionnaire with eight fields of daily activities, such as ability to use a telephone or handle medication. The scale scores ranged among the participants from 0 (inability) to 8 (high ability) and they were classified into two categories: 0-6 and 7-8.

A modified version of the Functional Co-morbidity Index (FCI) was used to determine comorbidities ${ }^{20}$. The FCI included medical diagnoses of arthritis (rheumatoid arthritis and other connective tissue disorders), osteoporosis, asthma/chronic obstructive pulmonary disease, coronary 
artery disease, congestive heart failure, myocardial infarction, Parkinson's disease, stroke, diabetes mellitus, depressive symptoms, visual impairment, hearing impairment, and obesity (body mass index $>30$ ). Each of these diagnoses was given a numeric value of 1 and they were summed together. Diagnoses were determined from the information obtained in the comprehensive geriatric assessment, from medical records or from the Finnish Special Reimbursement Registers maintained by the Social Insurance Institution of Finland.

\section{Statistical methods}

In this study, Poisson regression models with robust error variance ${ }^{23}$ were used to estimate relative risk (RR) with a 95\% confidence interval (CI 95\%). All the models were adjusted for age, gender, education, diabetes and rheumatoid disease, and the Functional Comorbidity Index. Three additional sets of models were further adjusted for the total number of drugs, use of antihypertensives, and sedative load. All the statistical analyses were done using SPSS 22.0 software for Windows ${ }^{24}$.

\section{$\underline{\text { Results }}$}

The socio-demographic and health-related characteristics of the study population are presented in Table 1 and the unadjusted relative risks of the explanatory variables are in Table 2. The adjusted relative risks from the multivariate models are presented in Table 3. In this study population, the ADS scores ranged between $0-9$ and about $51 \%$ of the participants $(n=77)$ had no anticholinergic burden (ADS 0), $35 \%(n=53)$ had a moderate anticholinergic burden (ADS $1-2)$, and $14 \%$ of the participants $(n=22)$ had a high anticholinergic burden (ADS $\geq 3$ ).

The analyses showed that after adjusting for confounding factors (age, gender, education, diabetes, rheumatic diseases and FCI), participants with a high anticholinergic burden (ADS $\geq 3$ ) were more likely to have xerostomia (RR: 3.17; CI: 1.44-6.94) than participants without an anticholinergic burden (ADS 0). While participants with a moderate anticholinergic burden (ADS 1-2) also had a 
higher likelihood of xerostomia, the association was not statistically significant (RR: 1.27; CI: 0.57-2.85) compared to participants without an anticholinergic burden. When a continuous ADS was used, the relative risk was 1.20 (CI: 1.06-1.37) (Table 3).

After adjusting for confounding factors, participants with a high anticholinergic burden $(\geq 3)$ had a higher likelihood of low unstimulated salivary flow (<0.1 ml/min; RR: 2.31, CI: 1.21-4.41) compared to the reference group (ADS 0). The risk estimates for unstimulated salivary flow in the ADS 1-2 group varied between $0.55-2.14$. For a continuous ADS the relative risk was 1.22 (CI: 1.10-1.34) (Table 3).

Participants with either a moderate (ADS 1-2) or high anticholinergic burden (ADS $\geq 3$ ) were more likely — although not statistically significantly — to have low stimulated salivary flow $(<1.0 \mathrm{ml} / \mathrm{min}$; RR: 1.19, CI: 0.68-2.11 and RR: 1.49, CI: 0.79-2.79, respectively) compared to participants with an ADS score of 0 . When a continuous ADS was used, the relative risk was $1.09(0.98-1.20)$ (Table $3)$.

Additional adjustments for the total number of drugs, antihypertensives, and sedative load were also done for all the outcome variables. These analyses showed only a slight attenuation of the risk estimates for the ADS (Table 3, Models 2-4).

\section{$\underline{\text { Discussion }}$}

The results of the present study showed that a high anticholinergic burden was associated especially with low unstimulated salivary secretion and xerostomia and to a lesser extent with stimulated salivary secretion. These results are in line with earlier studies which have shown associations between anticholinergic burden ${ }^{11,12}$ or the use of multiple anticholinergic drugs ${ }^{28,29}$ and hyposalivation or xerostomia. The novelty of the present study is that the focus was on the association between the anticholinergic burden and dry mouth, measured with both hyposalivation and xerostomia. 
In this study population, both stimulated and unstimulated salivary secretions were measured and symptoms of xerostomia were asked during the interview. All three of these were used as outcome variables, which can be considered the most comprehensive method for assessing dry mouth ${ }^{1}$. This approach to defining dry mouth differs from most of the earlier studies, as only the study by Dessoutter et al..$^{28}$ included both unstimulated salivary secretion and questions about xerostomia as outcome variables. Other studies used either unstimulated salivary secretion, measured by a swab method $^{11}$, or xerostomia ${ }^{12,28,29}$ as an outcome variable. Furthermore, studies by Kersten et. $\mathrm{al}^{11}$ and Rudolph et al. ${ }^{12}$ had the main focus on the general anticholinergic adverse effects rather than on the dry mouth itself and they used the dry mouth just as a variable among the others to depict the peripheral adverse effects of anticholinergic drugs.

In the present study, the Anticholinergic Drug Scale (ADS) was used to measure anticholinergic burden, while earlier studies have used different methods to describe anticholinergic burden or the anticholinergic potential of the drugs used. Kersten et al. ${ }^{11}$ used the same measurement (ADS) as in this study, while Rudolph et al. ${ }^{12}$ used a different ranked list, the Anticholinergic Risk Scale (ARS), to measure anticholinergic burden. In studies by Günes et al. ${ }^{29}$ and Dessoutter et al. ${ }^{28}$, only the presence or intake of anticholinergic drugs was determined from the information in a pharmaceutical database or literature, such as Martindale or Theriaque. These studies did not take into account the total anticholinergic burden or variance in the anticholinergic potency of $\operatorname{drugs}^{28,29}$.

The abovementioned differences in research methods between the studies make direct comparison of the present findings with earlier results challenging. On the other hand, the earlier results lend support to the conception that anticholinergic drugs cause dry mouth, related to either hyposalivation or xerostomia. The findings of the present study can be seen to further support this conception by showing that the high overall anticholinergic burden was associated with unstimulated salivary secretion and xerostomia. 
When studying the effects of drugs on dry mouth, one must take polypharmacy into account. Previous studies have shown that simultaneous use of multiple drugs, regardless of class or type, can have an additive effect to cause dry mouth, especially xerostomia ${ }^{31-34}$. In contrast, our previous study from the same GeMS population ${ }^{34}$ showed that the number of drugs by itself is not an essential risk factor for hyposalivation, but the effects of the drugs are carried out by drugs with sedative properties. The present study also lends support to the assumption that the total number of drugs itself might not be a major risk factor for hyposalivation or xerostomia.

The analyses (Table 3, Model 2 \& 3) showed that the total number of drugs had the weakest association with all the outcome variables, the risk estimates varying between $0.86-1.10$, whereas sedative drugs were most strongly associated with stimulated salivary secretion, with the risk estimate for SL $\geq 3$ being 1.57 (0.89-2.75). On the other hand, the anticholinergic drugs had the strongest association with unstimulated salivary secretion and xerostomia. These findings are in line with our previous study, which showed similar results regarding the effects of drugs with sedative properties and the total number of drugs ${ }^{34}$. Because the adjustments for total number of drugs, antihypertensives, or sedative drugs caused only a slight attenuation of the relative risks, the effect of anticholinergic burden seems to appear to be independent of abovementioned factors.

\section{Measurment of dry mouth}

One of the strengths of the study was that both objective measure of salivary secretion and subjective measure of xerostomia were used as outcome variables. This approach is considered the most precise method of describing the dry mouth ${ }^{1}$. There are many studies which have studied an association between drugs and xerostomia, but at the same time, there is lack of studies which have focused also on the association between drugs and salivary secretion ${ }^{12}$.

\section{Measurement of anticholinergic burden}


The Anticholinergic Drug Scale (ADS) was used as a measure of anticholinergic burden and this enabled us to take into account the additive effect and variation in the anticholinergic potency. It adjusts the burden with the dosage of drugs and it has been proven to be a valid measure of anticholinergic burden among the older population, in both institutional ${ }^{8}$ and community settings ${ }^{35}$. The ADS includes 117 drugs with known anticholinergic activity ${ }^{8}$, whereas the other scales and metrics (e.g., the Anticholinergic Risk Scale or the Anticholinergic Cognitive Burden Scale) include fewer drugs ${ }^{12,36}$. Furthermore, the ADS covers 88 percent of the regularly used drugs in the study population ${ }^{16}$. Even though there is no standardized measurement of anticholinergic burden and discrepancies exist between the rating scales, the ADS has been proven to predict clinical outcomes with similar exactness as the other scales ${ }^{37}$.

There was on average a six-month time interval between the collection of drug information and clinical oral examinations and this should be taken in to account when interpreting the results. Yet, it is not unreasonable to assume that the time interval had only a minor influence on the results because of its short duration and because medication for chronic diseases is relatively permanent ${ }^{37}$. Another limitation related to drugs is the xerostomic effects, unrelated to anticholinergicy. The differentiating the main pathways of the xerostomic effect is difficult because the innervations of salivary glands are complex and it is not clearly known how concomitant use of multiple drugs effects on salivary glands at a receptor-level ${ }^{1}$. On the other hand, it is known that the most common way drugs cause dry mouth is by their anticholinergic effect and many commonly used drugs have anticholinergic effect ${ }^{4}$, meaning that xerostomic effects, not related to anticholinergicy, have only a minor influence on the results. This notion is further supported by the fact that additional adjustments for the total number of drugs, antihypertensives or drugs with sedative properties did not influence on the results significantly.

\section{Control of confounding}


Potential confounding factors such as age, gender, education, diabetes and rheumatoid diseases (the two most common diseases in the study population known to be associated with hyposalivation or xerostomia), and FCI were controlled for in the analyses. Also xerostomic effects of other drugs were controlled for by making models where the total number of drugs, antihypertensives, and drugs with sedative properties were used as covariates. Despite the effort to control for confounding factors, the possibility of residual confounding factors cannot be totally excluded. For example, anxiety $^{39}$ and stress ${ }^{40}$ have been shown to be associated with hyposalivation or xerostomia, but due to a lack of appropriate data, it was not possible to take these factors into account. Other factors associated with hyposalivation or xerostomia, such as depression, Parkinson's disease, HIV, Hepatitis $\mathrm{C}$ or radiotherapy of the head and neck region, were rare in these data.

Lastly, the original GeMS study was designed in such a way that the study population was homogeneous by ethnic background and place of residence. To increase the validity of this study, smokers and institutionalized participants were excluded. On the other hand, these exclusions decreased the size of the study population, which can be seen as a limitation.

\section{Practical implications}

In the older population, it is advisable to avoid prescribing excessively anticholinergic drugs because of the increased risk of severe adverse effects on both general health and oral health.

Patients with anticholinergic drugs should be offered guidance and information about proper oral hygiene and treatment of dry mouth; they should receive prophylaxis measures against oral diseases such as dental caries and oral candidosis; and they should have regular recalls for dental examinations.

\section{Conclusion}


A high anticholinergic burden was associated with low unstimulated salivary secretion and xerostomia.

\section{$\underline{\text { Acknowledgements }}$}

The GeMS study has been supported by the Social Insurance Institute and the City of Kuopio. A personal grant was given to Antti Tiisanoja by Finnish Dental Society Apollonia. We thank Paula Pesonen for her statistical guidance. 


\section{References}

1. Thomson WM. Dry mouth and older people. Aust Dent J 2015; 60 (Suppl): 54-63.

2. Wolff A, Zuk-Paz L, Kaplan I. Major salivary gland output differs between users and nonusers of specific medication categories. Gerodontology 2008; 25(4): 210-216.

3. Carnahan RM, Lund BC, Perry PJ et al. The concurrent use of anticholinergies and cholinesterase inhibitors: rare event or common practice? J Am Geriatr Soc 2004; 52(12): $2082-2087$.

4. Nishtala PS, Fois RA, McLachlan AJ, Bell JS, Kelly PJ, Chen TF. Anticholinergic activity of commonly prescribed medications and neuropsychiatric adverse events in older people. $J$ Clin Pharmacol 2009; 49(10): 1176-1184.

5. Ness J, Hoth A, Barnett MJ, Shorr RI, Kaboli PJ. Anticholinergic medications in community-dwelling older veterans: prevalence of anticholinergic symptoms, symptom burden and adverse drug events. Am J Geriatr Pharmacother 2006; 4(1): 41-51.

6. Kemper RF, Steiner V, Hicks B, Pierce L, Iwuagwu C. Anticholinergic medications: use among older adults with memory problems. J Gerontol Nurs 2007; 33(1): 21-29.

7. Low LF, Anstey KJ, Sachdev P. Use of medications with anticholinergic properties and cognitive function in a young-old community sample. Int J Geriatr Psychiatry 2009; 24 (6): $578-584$.

8. Han L, Agostini JV, Allore HG. Cumulative anticholinergic exposure is associated with poor memory and executive function in older men. J Am Geriatr Soc 2008; 56(12): 22032210.

9. Tune LE, Strauss ME, Lew MF, Breitlingeer E, Coyle JT. Serum levels of anticholinergic drugs and impaired recent memory in chronic schizophrenic patients. Am J Psychiatry 1982; 139(11): 1460-1462. 
10. Salahudeen M, Dufful S, Nistala P. Anticholinergic burden quantified by anticholinergic risk scales and adverse outcome in older people: a systemic review. BMC Geriatrics 2015; 15: 13. doi: 10.1186/s12877-015-0029-9.

11. Carnahan RM, Lund BC, Perry PJ, Pollock BG, Culp KR. The Anticholinergic Drug Scale as a measure of drug-related anticholinergic burden: associations with serum anticholinergic activity. J Clin Pharmacol. 2006; 46(12): 1481-1486.

12. Villa A, Wolff A, Narayana N, et al. World Workshop on Oral Medicine VI: a systematic review of medication-induced salivary gland dysfunction. Oral Dis 2016; 22(5): 365-382.

13. Miranda-Rius J, Brunet-Llobet L, Lahor-Soler E, Farré M. Salivary Secretory Disorders, Inducing Drugs, and Clinical Management. Int J Med Sci 2015; 12(10): 811-824.

14. Kersten H, Molden E, Willumsen T, Engedal K, Bruun Wyller T. Higher anticholinergic drug scale (ADS) scores are associated with peripheral but not cognitive markers of cholinergic blockade. Cross sectional data from 21 Norwegian nursing homes. Br J Clin Pharmacol 2013; 75(3):842-849.

15. Rudolph JL, Salow MJ, Angelini MC,McGlinchey RE. The anticholinergic risk scale and anticholinergic adverse effect in older persons. Arch Intern Med 2008; 168(5): 508-513.

16. Lampela P, Lavikainen P, Garcia-Horsman JA, Bell JS, Huupponen R, Hartikainen S. Anticholinergic drug use, serum anticholinergic activity, and adverse drug events among older people: a population-based study. Drugs Aging 2013; 30(5): 321-330.

17. Komulainen K, Ylöstalo P, Syrjälä AM et al. Determinants for preventive oral health care need among community-dwelling older people: a population-based study. Spec Care Dentist 2014; 34(1): 19-26.

18. Tiisanoja A, Syrjälä AM, Komulainen K et al. Sedative load, carious teeth and infection in the periodontium among community-dwelling older people [published online ahead of print November 27, 2015]. Gerodontology. doi: 10.1111/ger.12217. 
19. Navazesh M. Methods for collecting saliva. Ann N Y Acad Sci 1993; 20: 72-77.

20. Flink H, Bergdahl M, Tegelberg A, Rosenblad A, Lagerlo F. Prevalence of hyposalivation in relation to general health, body mass index and remaining teeth in different age groups of adults. Community Dent Oral Epidemiol 2008; 36(6): 523-531.

21. Thomson WM, Brown R, Williams S. Medication and perception of dry mouth in a population of institutionalised elderly people. $N Z$ Med J 1993; 106(957): 219-221.

22. Lawton MP, Brody EM. Assessment of older people: self-maintaining and instrumental activities of daily living. Gerontologist 1969; 9(3): 179-186.

23. Groll DL, To T, Bombardier C, Wright JG. The development of a comorbidity index with physical function as the outcome. J Clin Epidemiol 2005; 58(8): 595-602.

24. Linjakumpu T, Hartikainen S, Klaukka T, Koponen H, Kivelä SL, Isoaho R. A model to classify the sedative load of drugs. Int J Geriatr Psychiatry 2003; 18(6): 542-544.

25. Taipale HT, Bell JS, Uusi-Kokko M, Lönnroos E, Sulkava R, Hartikainen S. Sedative load among community-dwelling people aged 75 years and older: a population-based study. Drugs Aging 2011; 28(11): 913-925.

26. Zou G. A modified Poisson Regression approach to prospective studies with binary data. Am J Epidemiol 2004; 159(7): 702-706.

27. SPSS Inc. SPSS 22.0 for Windows. 2013; 22.0.0.

28. Desoutter A, Soudain-Pineau M, Munsch F, Mauprivez C, Dufour T, Coeuriot JL. Xerostomia and medication: a cross-sectional study in long-term geriatric wards. $J$ Nutr Health Aging 2012; 16(5): 575-579.

29. Güneş Z, Denat Y, Müezzinoğlu M, Sen S, Yılmaz S, Atlı E. The risk factors effecting the dry mouth in inpatients in Hospital in west Anatolia. J Clin Nurs 2012; 21(3-4): 408-414. 
30. Bardow A, Nyvad B, Nauntofte B. Relationships between medication intake, complaints of dry mouth, salivary flow rate and composition, and the rate of tooth demineralization in situ. Arch Oral Biol 2001; 46(5):413-423.

31. Liu B, Dion MR, Jurasic MM, Gibson G, Jones JA. Xerostomia and salivary hypofunction in vulnerable elders: prevalence and etiology. Oral Surg Oral Med Oral Pathol Oral Radiol 2012; 114(1): 52-60.

32. Viljakainen S, Nykänen I, Ahonen R et al. Xerostomia among older home care clients. Community Dent Oral Epidemiol 2016; 44(3): 232-238.

33. Villa A, Abati S. Risk factors and symptoms associated with xerostomia: a cross-sectional study. Aust Dent J 2011: 56(3): 290-295.

34. Tiisanoja A, Syrjälä AM, Komulainen K et al. Sedative load and salivary secretion and xerostomia in community-dwelling older people. Gerodontology 2016; 33(2): 177-184.

35. Ness J, Hoth A, Barnett MJ, Shorr RI, Kaboli PJ. Anticholinergic medications in community-dwelling older veterans: prevalence of anticholinergic symptoms, symptom burden and adverse drug events. Am J Geriatr Pharmacother 2006: 4(1): 41-51.

36. Boustani M, Campbell N, Munger S, Maidment I, Fox C. Impact of anticholinergics on the aging brain: a review and practical application. Aging Health 2008; 4(3): 311-320.

37. Marcum Z, Hanlon J, Murray M. Improving Medication Adherence and Health Outcomes in Older Adults: An Evidence-Based Review of Randomized Controlled Trials. Drugs Aging. 2017; 34(3): 191-201.

38. Bergdahl M, Bergdahl J. Low unstimulated salivary flow and subjective oral dryness: association with medication, anxiety, depression, and stress. J Dent Res 2000; 79(9): 1652_ 1658. 
39. Hugo FN, Hilgert JB, Corso S, Padilha DM, Bozzetti MC, Bandeira DR et al. Association of chronic stress, depression symptoms and cortisol with low saliva flow in a sample of south-Brazilians aged 50 years and older. Gerodontology 2008; 25(1): 18-25. 